\title{
LA FILOSOFÍA SIN MUNDO DE EMMANUEL LÉVINAS
}

\author{
Claudia Gutiérrez* \\ Universidad de Chile \\ calacello@hotmail.com
}

\begin{abstract}
Resumen
Como marca y vaticinio de un pensamiento por venir, el gesto que inaugura en $1935 \mathrm{el}$ pensamiento de Lévinas consiste en la operación de una verdadera epojé mundana, que decanta, implícitamente, del examen de la vieja noción de evasión. Este trabajo intentará hacer explícita dicha epojé, para luego desentrañar las apuestas y destinos teóricos posibles para un punto de partida no-mundano para la obra de Lévinas.
\end{abstract}

Palabras clave: mundo, evasión, Ser, rostro.

\section{Abstract}

As note and prophecy of a coming thought, the opening sign of Emmanuel Lévinas' thought in 1935 consisted in the operation of a truly worldling epojé, which is implicitly decanted out of review of the old notion of evasion. This paper aims to make explicit the notion of wordling epoje, in order to disembowel the possible theoretical issues and directions that this concept may follow, thus establishing a non-worldlind starting point for the work of Lévinas.

KEY WORDS: world, evasion, being, face.

$\overline{\mathrm{R} A}$

El siguiente texto intenta explorar el pensamiento de Lévinas a la luz de la cuestión del "mundo", proponiendo en ello una clave de lectura posible, capaz de abrir el texto levinasiano desde otros lugares. En efecto, la cuestión del "mundo" urde gran parte de la meditación levinasiana, cuyos matices declinan, aunque en sordina, los acentos éticos y políticos de la misma. Indagando la clave "mundana", intentaremos al mismo tiempo no solo desagregarnos explícitamente del vasto prontuario de lecturas éticas del pensamiento levinasiano, sino además, procurar nuevos significantes para comprender los lineamientos éticos mismos que recorren evidentemente la obra de Lévinas.

¿Una filosofía sin mundo? Desde sus inicios en 1935, la filosofía de Emmanuel Lévinas se inaugura con un gesto especulativo decisivo, que consiste en la puesta

Este artículo forma parte del proyecto de Post-doctorado Fondecyt 3090011. Departamento de Filosofía, Universidad de Chile. 
fuera de juego de la alternativa del mundo. Se trata de una especie de epojé mundana -operada implícitamente- cuyo sentido puede traducirse como la refutación del horizonte mundano como origen y garante del sentido, esto es, como estructura ontológicoexistencial donde se jugaría el sentido primero de lo humano. En efecto, el origen del sentido, el "sentido del sentido", según una clásica expresión levinasiana, reside en la exploración de una alternativa extra-mundana. El rostro del otro, dirá Lévinas, "no es des-cubrimiento de un mundo" (Lévinas 2001, p. 270), él es estrictamente ausencia de mundo. ¿Pero en qué sentido hemos de entender esta ausencia de mundo, esta extramundanidad? ¿Acaso la filosofía de Lévinas no se interesa por las cuestiones que suceden en el mundo? ¿Cuáles son los límites y fecundidades de un punto de partida no-mundano para el filosofar? ¿De qué manera un gesto especulativo que arranca de lo sin-mundo puede luego concretizarse mundanamente? Por otra parte, esta especie de epojé mundana inaugural que intentaremos explicitar va a la par con el trabajo de la noción de "evasión", verdadera gestora, según nosotros, de la impronta extra-mundana de la filosofía de Lévinas.

En este trabajo procederemos de la manera siguiente:

En primer lugar, examinaremos algunos de los puntos fundamentales del texto de 1935, De l'évasion (Lévinas 1982) e intentaremos despejar las apuestas teóricas de la noción de evasión.

En segundo lugar, nos referiremos a la perspectiva no mundana que la noción de evasión determina, examinando uno de los conceptos ejemplares que se descifra cabalmente bajo esta epojé mundana: el concepto de rostro.

De la evasión a la epojé mundana

Es en 1935, con el texto De l'évasion, y con una fuerza teórica original, que la categoría de evasión aparece en la meditación levinasiana. El campo reflexivo que ella inaugura es determinante en lo que concierne la vieja cuestión del Ser y las determinaciones ónticas y ontológicas de esta última. A este respecto, afirmemos desde ya lo siguiente: en la reflexión levinasiana, la primera relación comprensiva con el ser del sujeto no considera la elucidación de la estructura ontológica "estar-en-el mundo" (In-der-Weltsein), estructura que como sabemos supone una estancia dada de antemano para el ente privilegiado que es el sujeto, a partir de la cual podría trazar y pro-yectar todas sus aventuras ontológicas. En Lévinas, más que de permanecer, o de habitar, de lo que se trata es de salir. Tal es la divisa especulativa que guía las reflexiones de esta época. ¿Salir? ¿De qué lugar? ¿Y para dirigirse a dónde?

Para un pensamiento atento a lo que piensa y a las modalidades de cómo surge lo pensado, las circunstancias históricas condicionan de una manera radical el ejercicio del pensamiento. El horizonte conceptual y comprensivo del texto De l'évasion surge 
así, y por una parte, haciéndose cargo de la pesadez ambiental de la guerra que se anunciaba, e intentando, por otra parte, ya adentrarse en la re-comprensión y reescritura de conceptos operatorios de gran parte de la historia de la filosofía. Es bajo esta doble exigencia comprensiva que ha de descifrarse el sentido último del texto De l'évasion.

Si De l'évasion pone al centro del debate la cuestión de la salida del ser -"se trata de salir del ser" (Lévinas 1982, p. 127)- correlativamente la suspensión de la estructura mundana como condición de posibilidad y evidencia para la ordenación del sentido para lo ente surge como su corolario fundamental. La filosofía sin mundo de Lévinas nace aquí.

Examinemos el texto De l'évasion y enunciemos brevemente los ejes argumentativos mayores que nos conducen, según nosotros, a la comprensión de la noción de evasión y posteriormente a su corolario no-mundano. Distinguiremos cuatro: la crítica al idealismo y a la posición de sujeto; contra el idealismo, la renovación de la cuestión del "ser en tanto ser"; la reformulación y crítica de la cuestión del ser; la necesidad de la evasión.

Primero, la crítica al idealismo: Lévinas critica la posición de suficiencia, de autarquía del sujeto que resume la figura del cogito y la conciencia. Según Lévinas, para el sujeto cognoscente del idealismo, la salida al mundo se hace innecesaria. Todo se resuelve desde la posición del ego, que clausurado en sus propios límites de ego no es sino clausura de la apertura al horizonte del mundo. Se trata así de la primacía del "Yo pienso" por sobre el Ser y la existencia. La objeción mayor de Lévinas a la perspectiva idealista podemos resumirla así: ¿Cómo sale el ego de sí mismo para alcanzar el objeto? Porque el idealismo, según Lévinas, no critica la existencia misma del cogito (él está ocupado en comprender y definir su esencia) se hace necesario renovar la antigua cuestión del Ser.

Segundo, renovación de la cuestión del "ser en tanto ser": contra la posición idealista, y en un gesto posiblemente tributario del Heidegger de Ser y Tiempo, Lévinas se da como tarea interrogar la existencia concreta del sujeto. Porque se trata de interrogar la existencia y no la esencia o la naturaleza de lo que es, en esta perspectiva la existencia del sujeto se descubre en un anclaje existencial infranqueable. Dicho anclaje podría ser leído siguiendo aquí la apuesta de lectura de Jacques Rolland ${ }^{1}$, lector de Lévinas, según la cual la existencia del sujeto se revela arrojada en el mundo, a la manera de una singular Geworfenheit, y en este sentido preciso ella se revela trascendiendo en permanencia al ego.

Tercero, reformulación y crítica de la cuestión del ser: en un segundo momento, reformulando la pregunta por el ser, el "ser en tanto ser" (existencia de lo que es), una particular acepción de la Geworfenheit guiaría estas cuestiones ontológicas. En esta lectura, todo pasa como si Lévinas insistiera en el estar-arrojado, olvidando completamente

Remitimos aquí al texto escrito por Rolland y que sirvió de introducción a la reedición de De l'évasion en 1982: "Sortir de l'être par une nouvelle voie". 
(y de ahí su singularidad) el momento del Entwurf (proyecto), que como sabemos es necesario para la cabal comprensión de la disposición afectiva del Dasein.

Cuarto, la necesidad de evasión: un análisis existencial del sujeto encarnado, anclado en una Geworfenheit sin remisión posible a la proyectualidad de su existencia, permite el posicionamiento de una "necesidad de evasión". Esta especie de "nueva ontología" levinasiana, hija herética de la ontología heideggeriana, revela así una noción de Ser que se describe como plenitud del Ser o de la existencia y no así como carencia u ocultación del mismo. Insistiendo en lo irrecusable de la existencia encarnada como exposición plena a nuestro hecho de existir, Lévinas comprenderá esta "necesidad de evasión" como evasión del Ser.

Detengámonos en los puntos tercero y cuarto. En la introducción que hiciera Jacques Rolland para la reedición en 1982 de De l'évasion, encontramos la clave interpretativa que guía nuestra reflexión. Rolland escribe:

Se podría decir que la reflexión de Lévinas va a detenerse en la Geworfenheit con el objeto de descubrir y describir una situación en la que la existencia no encuentra en ella una propensión a ir más allá de la situación impuesta, una situación en la que el estar-arrojado paraliza de alguna manera toda posibilidad de proyectarse (Lévinas 1982, p. 28).

Esta detención en la Geworfenheit va a conducir a una disociación del otro momento decisivo para la comprensión del ser del Dasein, a saber, el momento del proyecto (Entwurf), tal es la tesis de Rolland. Quisiéramos profundizar en esta particular hermenéutica de la facticidad y hacer surgir el siguiente corolario: una de las consecuencias mayores de esta desunión, producto de la permanencia insistente en la Geworfenheit es precisamente la obliteración de la estructura mundana. La hermenéutica de la facticidad levinasiana, por así llamarla, -y en esto se marca un distanciamiento con Heidegger- conduce a un plano especulativo donde la cuestión del mundo está ausente. ¿Cuál es el sentido de este estar-arrojado?

La especificidad y uso de la Geworfenheit en Lévinas dice relación con una insistencia, una iteración en el carácter de ese "estar-arrojado" del hombre, al punto de constituirse este arrojamiento mismo en una especie de fardo existencial. Como si la existencia tradujese una condenación a ser. Imposible de sustraerse a la Geworfenheit, el hombre se descubre como un ser encadenado a sí, un "être rivé". Porque "El hecho de existir se refiere sólo a sí" (Lévinas 1982, p. 100), correlativamente una ausencia de devenir surge como su consecuencia primera.

Lo distintivo de este particular "estar arrojado" del sujeto dice relación con el hecho de que la existencia se descubre al mismo tiempo como una existencia encarnada. Esto es, y a diferencia de Heidegger, el Ser-ahí levinasiano (por llamarlo de alguna manera) dispone de cuerpo, él es fundamentalmente un nudo sintiente. Esta concepción de sujeto encarnado permite así los análisis del sufrimiento, la náusea, la vergüenza, el placer, el malestar, etc., que nutren el texto De l'évasion. Todas estas experiencias que involucran el cuerpo descubren la presencia del ser como $m i$ ser, sellando así la existencia encarnada no solo como una instancia primordial, estructurante de la subjetividad, sino 
y por esto mismo, como un hecho intransferible. Se trata, de este modo, de un anclaje existencial sin posibilidad de futuro, "sans-issue" (sin salida), dice Lévinas, en el que la existencia se hace dramática y sin resguardo posible a partir de sí-mismo. De ahí la necesidad de evasión, es decir, necesidad "de romper el encadenamiento más radical, más irremisible, el hecho que el yo es uno-mismo (...)" (Lévinas 1982, p. 98).

Siguiendo el texto De l'évasion, podremos distinguir dos figuras de evasión: evasión de lo ente y evasión del ser. Es en torno a esta última, y en oposición a la primera, que se juega la apuesta original de Lévinas. Por cuestiones de extensión, no podremos referirnos aquí al sentido de la evasión de lo ente. Baste con decir que en el análisis levinasiano la primera figura de evasión - que es un motivo literario propio a los románticos del siglo XVIII y XIX-permanecería aún inscrita en la lógica del ser, como si la "necesidad de evasión" no tradujese más que el anhelo de una realidad superior y mejor, que vendría a subsanar una falta, una falla, a la manera de un paraíso perdido o de una proyección utópica. A este respecto, Lévinas escribe: "La insuficiencia de la condición humana nunca ha sido comprendida de otro modo que como una limitación del ser [...]" (Lévinas 1982, p. 93). Así, si esta necesidad de evasión traduce un deseo de trascendencia, ella es tal por carencia o limitación de Ser. Esta cuestión es clave en la obra posterior de Lévinas, pues si la trascendencia tiene un sentido, éste no puede estar sino fuera de la lógica del ser².

La apuesta de Lévinas es radicalmente otra. No se trata de una necesidad de evasión de lo ente sino del Ser. Un análisis de la noción de "necesidad" permitirá posicionar mejor esta distinción. En efecto, si corrientemente "necesidad" alude a aquello que nos falta, a lo que carecemos, el sentido de "necesidad" que propone Lévinas no comprende una figura de carencia sino de exceso. En efecto, la necesidad de evasión traduce un deseo de trascendencia, no por carencia sino por exceso de ser. Veamos.

Según Lévinas, "La insuficiencia de la condición humana nunca ha sido comprendida de otro modo que como una limitación del ser, sin que nunca haya sido considerada la significación del 'ser finito" (Lévinas 1982, p. 93). Para Lévinas, la cuestión se juega precisamente en la trama del "ser finito", de lo humano y del "otro hombre". Considerar la significación del "ser finito" en tanto finito, sin recurrir por ello a ningún otro concepto que venga a alumbrar el sentido de esta significación, tal es la intención especulativa de Lévinas. En esta perspectiva, el ser finito que es el sujeto no

2 Esta figura de evasión de lo ente que implica la proyección de un lugar mejor, traduce, por cierto, un anhelo de trascendencia, una búsqueda de lo otro. No obstante, tratándose de la salida de lo ente que aspira a una realidad no defectuosa de ser, el destino de esta salida permanece circunscrita en los límites del ser. Por consiguiente, es la noción misma de "trascendencia" que se yergue y cobra sentido bajo la sombra del ser, cuestión inconcebible en la meditación levinasiana, en la que el sentido de la trascendencia se alza más allá del ser, en lo que Lévinas comprende bajo la formula del de otro modo que ser. "Si la trascendencia tiene un sentido, no puede significar otra cosa, para el acontecimiento de ser-para el esse, para la esencia- que el hecho de pasar a lo otro que el ser" (Lévinas 1999, p. 13). 
podría concebirse en términos de una limitación de ser. Esto sería aun remitirlo a un sentido que lo contendría, sería remitirlo a un posible.

En el horizonte de un análisis de lo "finito en tanto finito" y ya a la luz de una Geworfenheit encarnada y sin porvenir, una distinción entre la existencia y la esencia de lo que es, aclara aún mejor el propósito de Lévinas. Solo un análisis en términos de la existencia de lo que es, que describa fenomenológicamente la existencia concreta del sujeto, fuera de todo esencialismo, podría hacerse cargo de una descripción de lo "finito en tanto finito". Estamos aquí ya en posición de distinguir la fisonomía de un estar-arrojado cuya particularidad traduce la agudeza de un presente irremisible. En este arrojamiento, la existencia aparece anclada a sí misma sin posibilidad de anticipación ni de futuro a partir de sí, en una palabra, sin horizonte. "La existencia es un absoluto que se afirma a sí mismo sin referirse a nada diferente" (Lévinas 1982, p. 98). Amarrada a un presente sin remisión, la existencia se nos presenta como una experiencia aguda de lo sin fin, de lo ilimitado. Como si el hecho de existir tradujese "una tara más profunda" (Lévinas 1982, p. 94). Una tara más profunda -y según el doble sentido de tara, peso y falla- que significa en la agudeza de este estararrojado una experiencia de lo sin límite, en el sentido de lo que no se acaba nunca, justamente por lo intransferible de la experiencia o de la existencia encarnada. Como la experiencia del dolor o de la vergüenza. "Lo que aparece en la vergüenza es por tanto precisamente el hecho de estar clavado a uno mismo, la imposibilidad radical de huir de sí para ocultarse de uno mismo, la presencia irremisible del yo a sí mismo" (Lévinas 1982, p. 113).

Se trata, en resumen, de la experiencia del ser ilimitado, de la existencia pura, del hay ser. En una palabra, experiencia de un exceso.

Ante tal escenario, para el sujeto ya no será cuestión de trascender el ser finito (lo que sería aún distinguir un punto de salida y un destino) sino de trascender el ser mismo. No se trata ya de trascender la existencia limitada sino la existencia misma. No se trata de salir de lo ente sino de salir del ser. En otras palabras, se trata de una evasión por exceso de ser. La necesidad de evasión por exceso de ser se hace así "necesidad de excedencia" (Lévinas 1982, p. 98). Curioso neologismo que sugiere ya un subir, un ascender (scando), pero a través de un gesto centrífugo inscrito en la preposición "ex" de ex-cedencia, en el que si bien es cierto conocemos el punto de partida, de salida, el destino de esta salida resulta indefinido.

La consecuencia teórica mayor de este gesto especulativo de la evasión por excedencia es la suspensión de la alternativa mundana como sostén para la constitución del sentido. Se trata de una verdadera epojé, cuya función es por sobre todo de orden metodológico, pues ella es determinante para la obra posterior de Lévinas. En efecto, esta epojé mundana suspende la lógica del lugar y las determinaciones espaciales como fondo o sostén donde acunan los horizontes de sentido capaces, o tenidos por tales, de describir lo humano. En la perspectiva levinasiana, el lugar de lo humano, es precisamente defección de todo correlato mundano, defección del espectáculo. 
Obliteración mundana. Rostro y extramundanidad

Hemos dicho que la especificidad y uso de la Geworfenheit en Lévinas traduce una insistencia, una iteración en el carácter de ese "estar arrojado" del hombre, al punto de constituirse este arrojamiento mismo en una suerte de "fardo" existencial. Al modo de una experiencia de lo "sin salida", condenado a ser, imposibilitado de sustraerse al destino de un presente encarnado que no deviene ni termina, el hombre se descubre irremediablemente disociado de sus posibles. Una de las consecuencias mayores de esta disociación es que en el mismo gesto lo que se suspende es la estructura del mundo, lo hemos dicho. Abandonando la dimensión de la proyectualidad, se abandona al mismo tiempo la alternativa del mundo. Para comprender esto, recurramos una última vez a Heidegger.

Como sabemos, en Heidegger la comprensión de la Geworfenheit va de la mano de la noción de Entwurf. Pero como también sabemos, la justa comprensión del proyecto, "lo que está lanzado hacia adelante" 3 , no encuentra asidero sino en esta otra estructura que define el ser del Dasein, y de partida, como un ser-posible. Esto es, el ser del Dasein no se juega en el plano de un puro poder cerrado sobre sí mismo, sin puertas ni ventanas, como una mónada; su ser se encuentra profundamente abierto a su devenir. Es éste el sentido del parágrafo §31 de Ser y Tiempo:

El Dasein no es algo que está-ahí y que tiene, por añadidura, la facultad de poder algo, sino que es primariamente un ser-posible. El Dasein es siempre lo que puede ser y en el modo de su posibilidad (...) el Dasein es un ser-posible entregado a sí mismo, es, de un extremo al otro, posibilidad arrojada (Heidegger 2002, p. 147).

Pero si consideramos que en cuanto arrojado, el Dasein comprende este arrojamiento como "modo de ser del proyectar" (Heidegger 2002, p. 148), este modo de ser no puede sino desplegarse sobre la estructura de "Estar-en-el-mundo" (In-der-Welt-sein), verdadera cuna existencial para el devenir del sujeto. Dicho de otro modo, el "estar lanzado hacia adelante", el hecho de que el sujeto sea un "ser-posible", supone así un horizonte portador de todos los posibles, un contexto capaz de acoger todos los proyectos: el mundo. En palabras de Heidegger: "El proyecto concierne siempre a la plena aperturidad del estar-en-el-mundo" (Heidegger 2002, p. 149). El panorama en Lévinas es radicalmente otro.

3 Citemos in extenso la nota de Rivera que refiere al Entwurf en Ser y Tiempo. “ ، ... proyecto...’' en alemán, Entwurf(destacado en el texto original). Esta palabra debe ser entendida en su sentido literal: pro-yecto significa lo que está lanzado hacia adelante. Y este "delante" debe ser entendido como un futuro. Comprender es esencialmente pro-yecto o "proyección", como traduce Gaos, porque en él (en el comprender) el Dasein se lanza hacia su futuro y abre ese futuro como posibilidad" (Heidegger 2002, p. 435). 
En efecto, insistiendo Lévinas en el "estar arrojado", sin remisión a ningún posible, deja de lado la idea que define al sujeto según esta propensión a ir más allá de él, este "anticiparse". Porque el Ser no deviene, la condición de sujeto en Lévinas no traduce un "ser-posible". Consiguientemente, la estructura mundana, cuna de todos los posibles, contexto y amparo de todo devenir, queda irremediablemente fuera del horizonte especulativo o, al menos, queda en suspenso. Reconocemos aquí la reducción fenomenológica, la epojé, que anima nuestra lectura.

¿Cuál es el sentido de esta epojé? El sentido de esta epojé mundana responde en primer lugar a una intención estratégica y metódica, que determinará los acentos éticos de la obra levinasiana posterior, cuestión ética que evidentemente excede el marco especulativo de De l'évasion. Operando dicha suspensión, Lévinas no solo pone al centro de la meditación la irreductibilidad del sujeto a una trama superior que lo contendría, sino además hace decantar una filosofía del no-lugar, de la no residencia, perspectiva clave en la fisonomía de la obra levinasiana. Porque el primer gesto filosófico no es un "habitar" sino un salir del mundo, la salida del Ser por excedencia abre la vía a una búsqueda de sentido del orden de la extraterritorialidad. Intentemos comprender un poco más esta línea de argumentación.

En el marco de una reducción mundana inicial, pues el mundo no puede ser garante de sentido, ni contexto de mis posibles, quedan instaladas por otra parte las bases para una producción de conceptos sin mundo. En esta producción conceptual extramundana, lo que Lévinas buscará es sentar la posibilidad de un nuevo espacio garante del sentido, en el que el sujeto pueda desentrañar su sentido último, y en este sentido primero. Uno de los conceptos ejemplares en esta perspectiva extramundana es el concepto de "rostro", que como sabemos y en clave levinasiana este rostro del otro es de entrada ético. La eticidad del rostro es estrictamente proporcional a lo no mundanidad que lo sostiene.

La especificidad del rostro dice relación con lo que Lévinas nombra una "defección de la fenomenalidad" (Lévinas 1999, p. 144). Contra la lógica del fenómeno que despunta a partir de un horizonte, donde tiempo y espacio garantizan su comparecer, la noción de rostro es una noción, digamos, catastrófica. ¿En qué sentido? Según el sentido etimológico de la palabra catástrofe, es decir como el momento del desenlace o clausura de una pieza teatral, de una tragedia. Pero "catástrofe" no es solo un desenlace o un fin, así sin más. Es desenlace en la medida en que ella es figura de alteración o trastorno de un relato. Una catástrofe es lo inesperado, lo impensado, lo que sucede sin nuestro consentimiento, pese a toda previsión. Como figura de lo inesperado, ella marca un desenlace cuya especificidad consiste en interrumpir. Ella altera una trama -temporal y espacial- al mismo tiempo que introduce otra. Bajo esta perspectiva, la determinación del rostro como catástrofe que aquí proponemos, traduce el hecho de un imposible (pues es impredecible), que irrumpe en nuestra trama propia.

El rostro es un imposible que irrumpe en el mundo; cierto, pero a partir de un lugar que está fuera del mundo. De ahí su poder de trastorno e irrupción. En este sentido, la noción catastrófica de rostro opera como una verdadera pantalla conceptual respecto 
de todo relato que no sea la irrupción misma. El poder de refracción es estrictamente proporcional a la posibilidad de irrupción.

La fuerza, digamos epistemológica, de la noción de rostro reside así en que ella refracta toda consideración mundana que pretendiera definir este mismo rostro. El rostro no es rostro sino porque es precisamente lo sin mundo por excelencia. A la manera de un signo roto, o como un perturbador significante sin significado, "desnudo de su propia imagen" (Lévinas 2001, p. 270), el rostro significa por sí mismo, esto es "independiente de esta significación recibida del mundo" (Lévinas 2001, p. 270). Pero ya antes que el mundo mismo, el rostro refracta toda definición plástica de este mismo rostro, como figura de una extrema pobreza plástica. Profundamente iconoclasta entonces, que perfora o destruye su propia imagen, eso es el rostro. "El rostro horada su propia esencia plástica" (Lévinas 2001, p. 270).

La desnudez extrema del rostro supone más profundamente la imposibilidad de un horizonte espectacular, es decir, supone la imposibilidad de una noción de lugar que sostenga el espectáculo del rostro, la exhibición o mostración del mismo. En este sentido específico, el rostro es ausencia y defección de mundo.

El rostro, dice Lévinas, es figura de una proximidad sin distancia, esto es, el rostro significa "supresión de la distancia” (Lévinas 1999, p. 142) ¿Qué quiere decir que el Otro como rostro sea próximo pero de una proximidad "sin distancia"? En primer lugar que el rostro que no puede ser sino rostro del Otro saca a este mismo Otro de toda consideración en términos espaciales. Es decir, el rostro como categoría no espacial no es susceptible de ninguna extensión. Contrariamente a la cosa que necesita del espacio, el rostro no es mensurable, no tiene límites, bordes, aristas o escorzos. Por esta razón, Lévinas podrá sin problema instalar una semántica de lo inconmensurable para definir el rostro. Rostro como exterioridad absoluta, como trascendencia, como infinito, etc., son tantas de las maneras de nombrar aquella inconmensurabilidad que supone la no espacialidad. Esta ausencia de distancia se comprende positivamente como una cercanía, como "proximidad" dice Lévinas. Esta proximidad sin espacio, esta proximidad radical, es decir, sin posibilidad de reculada, constituye toda la esencia de la cercanía ética. En segundo lugar, la "proximidad sin distancia" que aporta el rostro significa que lo no extenso de este rostro es expresión o figura de un no-lugar, o lo que es lo mismo, el rostro es ausencia de lugar. Si el rostro escapa a las determinaciones espaciales, él escapa al mismo tiempo a las determinaciones del lugar. Dicho de otro modo, si el "rostro" toma sentido en las articulaciones de una "proximidad sin distancia", el rostro se instaura en una ausencia de visión panorámica, él pone fuera de juego toda situación. Como in-situable entonces, la "fenomenalización" del rostro, por llamarla de alguna manera (“epifanía” del rostro dirá Lévinas), se produce en la inflexión de una especie de no-lugar. Es por esto, por la no-espacialidad del rostro, por la ausencia de horizonte que sostenga su "aparecer", que él es la interdicción misma de todo relato mundano, y es por esto también, digámoslo al paso, que la categoría que le conviene mejor es aquella de la excepción, “ex-cepción del rostro" (Lévinas 1999, p. 26), precisa Lévinas. Muchísimos son los pasajes en los que el Otro, anunciándose como rostro, se instala de entrada en esta semántica de la excepción, fuera de toda noción de correlación e inclusión. 
El Otro como rostro, escribe Lévinas, no nos viene solamente a partir de un contexto, sino sin mediación, él significa por sí mismo [...] presencia, abstracta, no integrada al mundo [...] el rostro no es entonces el des-cubrimiento de un mundo [...] es un despojamiento sin ningún ornamento cultural -una absolución- un desprendimiento [...] Él es "extra-ordinario" (Lévinas 2001, p. 270).

Porque el rostro no devela un mundo, él funciona exactamente como clausura a toda estructura de lo posible. En lugar de abrir un mundo, un "mundo posible", como diría Deleuze, el rostro ético cierra un mundo, oblitera, obstruye todo horizonte.

Vemos de este modo que la impronta extramundana del rostro se condice perfectamente con los márgenes de la epojé mundana, que teje desde 1935 la reflexión propia de Lévinas. Alternativa del no-lugar como garante del sentido, del "sentido del sentido", que la vieja noción de evasión ya dejaría prescrita. Salida del ser por evasión en la que se conjura la lógica del lugar, lugar como destino de esta evasión, es decir, no-lugar. La exploración acabada de esta alternativa nutre con creces los delineamientos éticos del vasto campo de reflexión en Lévinas, cuestión ética aquella que nuestro trabajo no explora ${ }^{4}$.

Abriendo este texto preguntábamos: ¿En qué sentido hemos de entender esta extra-mundanidad? ¿Acaso la filosofía de Lévinas no se interesa por las cuestiones que suceden en el mundo? ¿Cuáles son los límites y fecundidades de un punto de partida no-mundano para el filosofar? ¿De qué manera un gesto especulativo qua arranca de lo sin-mundo puede luego concretizarse mundanamente? Declarábamos también que estas interrogantes son relevantes para quienes intentamos comprender la obra, difícil, de Lévinas, explorando una alternativa hermenéutica que trascienda el reduccionismo ético al que esta obra se ve corrientemente expuesta, a la vez que dicha alternativa podría aportar a comprender de otro modo también las apuestas éticas de la obra levinasiana.

Decíamos así que esta epojé mundana en la que una noción como aquella de rostro resuena perfectamente, respondía según nosotros, y ante todo, a una intención de carácter metódico. Esto es, y en esto reside según nosotros su particular ejercicio fenomenológico, Lévinas parte de la no evidencia del mundo para remontarse a una situación en la que todas las evidencias no solo encontrarían su punto de partida, sino además a la cual todas ellas habrían de ser remitidas: la intersubjetividad radicalmente asimétrica. Pero este punto de partida no-mundano para el ejercicio filosófico es solo eso, un punto de partida, cuya trayectoria no se cierra en la exclusión definitiva del mundo. Suspender el mundo en un primer tiempo, para recobrarlo luego en los intersticios de una intersubjetividad fundante. Tal sería a nuestro juicio la intención de

4 Bajo este precepto del no-lugar se descifra la clave ética del "de otro modo de ser" levinasiano, en el que ya no es cuestión de evasión del ser, sino de un desgarro de la esencia. "Se trata de pensar la posibilidad de un desgarro [arrachement] de la esencia. ¿Para ir adónde? ¿Para ir a qué región? ¿Para mantenerse en qué plano ontológico? Pero el desgarro de la esencia contesta el privilegio incondicional de la pregunta: a dónde. Significa el no-lugar" (Lévinas 1999, p. 21). 
Lévinas, perceptible a lo largo de su obra. Es por esto que la noción de "evasión" más que abandono del mundo es solo suspensión, epojé. En otros términos, la alternativa extramundana no es más que un eslabón en la secuencia especulativa de Lévinas. En dicha secuencia la vuelta al mundo se hace necesaria. En definitiva, se tratará entonces de una progresión metódica desde lo no-mundano, corolario implícito en la evasión del Ser hacia lo mundano.

¿Volver al mundo? ¿Es que el rostro puede devenir mundano? En efecto, porque el rostro ético no satisface ni agota todas las dimensiones de la vida humana ${ }^{5}$ es que una incrustación mundana se hace necesaria. Se trata, de esta manera, de aportar un contexto o un horizonte al rostro, lo que significa insertarlo en una dimensión en la que todas las situaciones -culturales, sociales, políticas, lingüísticas, sexuales etc.,comiencen a contar. Los alcances de esta inserción mundana producen una suerte de des-clausura de todas esas dimensiones hasta ahora relegadas o, mejor dicho, puestas entre paréntesis. Des-clausura ética del rostro podríamos llamar este movimiento, en que la dimensión ética entra en contrapunto con otras dimensiones. Es esto lo que Lévinas llama "dé-visager" el rostro (visage), esto es, hacer que lo no-figurativo del rostro se limite justamente como figura y se decline según un contexto; "des-figurar" sus límites éticos con el fin de dar figura a otras formas del mismo. En una palabra, se trata de hacer que su alteridad se haga "relativa a" "contigua a". Bajo este precepto mundano, la relación intersubjetiva ética profundamente asimétrica en Lévinas, deslinda los contornos de una necesidad de simetría intersubjetiva que garantice la concreción de las relaciones en el mundo. Que nos baste solo con anunciar dos direcciones ejemplares de esta necesidad de simetría o de proceso de inserción mundana del rostro: en primer lugar, los análisis contenidos en la sección "Fenomenología del Eros", de Totalidad e Infinito, en particular aquellos sobre la caricia, en los que el rostro se encarna y deviene sexuado. En segundo lugar, los análisis en torno a la figura del tercero, llave maestra para la inserción política del otro, en los que el rostro se "des-figura" y es llamado a ser parte de la comunidad ${ }^{6}$.

En una palabra, insertar el rostro en el mundo significa limitarlo, poner fin a la catástrofe, restituyéndolo a un horizonte de contigüidad y contemporaneidad. Especie de corrección de la ausencia de mundo inicial, en la que corregir significa concretamente traer a presencia mundana lo que de suyo no lo es: la infinición o inconmensurabilidad del rostro. Como rostro en el mundo, el rostro pierde su calidad de rostro abstracto y se hace concreto: se viste, se ornamenta, se contextualiza, se sitúa, dispone de idioma, se hace referente a un lugar, deviene ciudadano. En resumen, se localiza y se hace espacial, justamente se instaura como rostro en-el-mundo.

5 Para Lévinas el otro es también "individuo de un orden lógico o ciudadano de un Estado" (Adert et Aeschlimann 1989, p. 11).

6 "La relación con el tercero es una incesante corrección de la asimetría de la proximidad en la que el rostro se des-figura [dé-visage]" (Lévinas 1999, p. 246). 
Más que referirnos a una posible concepción de mundo en Lévinas, hemos intentado descubrir una filosofía sin mundo acentuando el carácter metódico de este gesto, la epojé. Abandono del mundo o ausencia estratégica entonces, que marca un ordenamiento singular para la filosofía de nuestro autor, ausencia destinada no obstante a ser recobrada y reconquistada con otros bríos. Reconquista mundana que por cuestiones de extensión este trabajo no deja más que anunciada.

Epojé mundana apenas perceptible en el ensayo de 1935. Salir del ser por una nueva vía, concluía provocadoramente Lévinas en este ensayo, vía insólita aquella que, en sordina, renuncia a la supuesta evidencia de los andamios en los que la existencia transcurre: el mundo, arriesgando temerariamente "invertir algunas nociones que al sentido común y a la sabiduría de las naciones les parecen las más evidentes" (Lévinas 1982, p. 127).

\section{Referencias bibliográficas}

Adert Laurent et Aeschlimann Jean-Christophe (1989), "Entretien”, en Aeschlimann Jean-Christophe, ed., Répondre d'autrui Emmanuel Lévinas. Suisse: Editions de la Baconnière, pp. 9-16.

Heidegger Martín (2002), Ser y Tiempo, traducción, prólogo y notas de Jorge Eduardo Rivera. Santiago: Universitaria.

Lévinas Emmanuel (1982), De l'évasion. Introduit et annoté par Jacques Rolland. Montpellier: Fata Morgana.

Vrin.

(2001), En découvrant l'existence avec Husserl et Heidegger. Paris:

(1990), Totalité et infini. Essai sur l'extériorité. Paris: Poche Essais.

(1999), Autrement qu'être ou au-delà de l'essence. Paris: Poche Essais. 